\title{
Design of MEMS Capacitive Pressure Sensor for Continuous Blood Pressure Monitor
}

\author{
Diem N. Ho ${ }^{1+}$, Ngoc-Hanh Dang ${ }^{2}$ and Vinh Q. Nguyen ${ }^{2}$ \\ ${ }^{1}$ Faculty of Computer Engineering, University of Information Technology - VNU-HCM, Vietnam \\ ${ }^{2}$ Faculty of Electrical and Electronics Engineering, Ho Chi Minh City University of Technology - VNU- \\ HCM, Vietnam
}

\begin{abstract}
The MEMS (Microelectromechanical System) pressure sensor has been investigated and manufactured into many commercial products; however, the commercial sensor design is not disclosed by the manufacturers. This paper presents a set of new design parameters for a capacitive pressure sensor which is aimed at self-manufacturing. This sensor design is capable to be used in a human blood pressure monitor for continuous measurement. The pressure range to be measured is from $0 \mathrm{KPa}$ to $50 \mathrm{KPa}$ which is in the range of human blood pressure. Design parameters are analyzed and simulated using COMSOL software. The pressure sensor of square diaphragm with edge dimension $1000 \mu \mathrm{m}$, thickness $15 \mu \mathrm{m}$, the gap between plates $3 \mu \mathrm{m}$, made of polysilicon is shown to have the best sensitivity for the applied pressure.
\end{abstract}

Keywords: MEMS, capacitive pressure sensor.

\section{Introduction}

MEMS pressure sensor has many advantages compared to conventional pressure sensor due to its low weight, low cost, reliable, smart function and occupies less space. Many commercial pressure sensors use piezoresistive sensing technique [1,2]. Piezoresistive pressure sensor is preferred because the properties of silicon material have been fully defined and the current silicon foundry facility can be used for mass production. Pieoresistive sensor has high coefficient of measurement but it also has high temperature coefficient. This limits the operating temperature and requires temperature compensation circuit. In spite of complex signal conditioning circuit, capacitive pressure sensor occupies more advantages over the piezoresistive one: low power consumption, low noise, high sensitivity, less affected by temperature, and good long-term stability [3, 4]. Advances in silicon micromachining techniques have also helped in the miniaturization of the capacitive pressure sensors. With small size and low energy consumption, capacitive pressure sensor fits for medical application, especially for the devices implanted in the body or be used consistently in a long time.

Continuous blood pressure monitoring is important for people requiring frequent blood pressure measuring throughout the day, especially patients with hypertension risk. There are many investigations on invasive blood pressure monitor as in $[5,6]$ but a safe, reliable one for long-term use is not yet commercially available [7]. Approaches to make the measurement non-invasive and still capable to do a measurement constantly have been proposed in many designs $[8,9]$. The pressure sensor in this work aims to operate in a non-invasive blood pressure measuring device such as a device at the wrist. The machine can measure continuous blood pressure, thus less power consumption is one of the essential characteristics of the device. That this device is worn at the wrist and can be frequently used demands a compact device. This work chooses to design the sensor of type capacitive pressure sensor regard to its advantages in medical application as mentioned earlier. This work will carry out an analysis on the centre deflection of sensor

+ Corresponding author. Tel.: (+84) 985300509.

E-mail address:diemhn@uit.edu.vn. 
diaphragm and capacitance sensitivity to point out the design parameters suitable to measure the human blood pressure in the range $0-50 \mathrm{KPa}$ (equivalent $0-375 \mathrm{mmHg}$ ).

\section{Capacitive Pressure Sensor Design}

\subsection{Sensor layout}

The sensor layout used in this paper is a formal capacitive pressure sensor, including two parallel plates acting as electrodes of a capacitor. The upper plate is usually moveable (diaphragm) and the lower one is fixed as illustrated in Figure 1. The thin diaphragm is held at a fixed potential. The diaphragm is separated from a ground plane by a chamber sealed under vacuum. External pressure applies on the diaphragm and move the upper electrode, resulting the capacitor between the plates will be changed. As a result, the capacitor changes are used for pressure sensing. The sensitivity of the sensor is related to the capacitive changes. More changes cause the higher sensitivity.

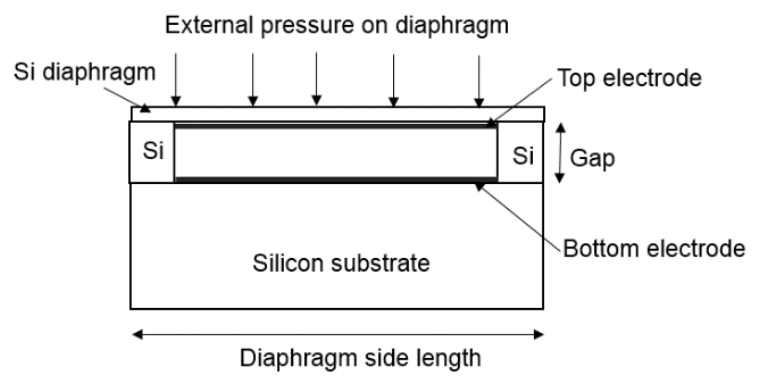

Fig. 1: Cross section of the capacitive pressure sensor.

Square and round diaphragms are frequently used in MEMS devices. It is also possible to use rectangular shape. But there is non-uniform stress distribution on rectangular diaphragm due to lack of symmetry of the structure, then decreasing the sensor sensitivity. Besides, no clear advantage between square and circular diaphragm with the same area under the same load is presented by Goodall [10]. Moreover, the silicon crystal structure has anisotropic nature, thus a square diaphragm can be fabricated more easily and accurately. As a result, it was decided to utilize a square diaphragm instead of a circular one.

\subsection{Mathematical analysis}

The governing differential equation for the deflection of thin plate is given by [11]:

$$
\frac{\partial^{2} w}{\partial x^{2}}+2 \frac{\partial^{2} w}{\partial x \partial y}+\frac{\partial^{2} w}{\partial y^{2}}=\frac{P}{D}
$$

where $w$ is the deflection of the diaphragm, $P$ is the applied pressure, $D$ is the flexural rigidity, given as:

$$
D=\frac{E h^{3}}{12\left(1-v^{2}\right)}
$$

by solving with boundary condition, where all sides of the sensor diaphragm are built in, that is:

$$
w=0, \frac{\partial w}{\partial n}=0
$$

where $n$ is the normal vector direction along the sides of the sensor diaphragm.

For square diaphragm, the maximum stress at the midpoint of each edge, and the maximum displacement of the diaphragm at the centre of the plate is as follow:

$$
\sigma_{\max }=\frac{0.308 P a^{2}}{h^{2}} ; \quad w_{\max }=-\frac{0.0138 P a^{4}}{E h^{3}}
$$

where $h$ is the diaphragm thickness, $a$ is the length of the square edge, $E$ is the Young's Modulus

The capacitance between two parallel electric conductive plates can be written as:

$$
C=\varepsilon_{0} \varepsilon_{r} \frac{A}{d}
$$


where $\mathrm{c}$ is capacitance, $\varepsilon_{0}$ is dielectric constant of vacuum, $\varepsilon_{r}$ is the dielectric constant of material. A is area of electrode plate and $\mathrm{d}$ is the gap between two electrode plate.

Based on Hooke's law, the change in thickness in the dielectric layer is proportional to the pressure change. As a result, the relationship between the applied pressure and the capacitance change can be expressed as Eqs.(6):

$$
\Delta d=d_{0} \frac{\Delta P}{E} ; \quad \Delta C=C_{0} \frac{\Delta P}{E-\Delta P}
$$

where $\Delta \mathrm{d}$ is thickness change of dielectric layer, $\mathrm{d} 0$ is original thickness of dielectric layer. $\Delta \mathrm{P}$ is applied external pressure, $\mathrm{C} 0$ is original capacitance when pressure is not applied and $\Delta \mathrm{C}$ is capacitance change when pressure is applied. The mathematical analysis is to verify the deflection of the diaphragm due to mechanically applied force and the capacitance between the diaphragm and the back plate.

\section{Results and Discussion}

The displacement and capacitance of the clamped square diaphragm will be modelled using COMSOL software. The COMSOL takes advantage of the symmetry in the geometry and models only a single quadrant of the device as shown in Figure 2.
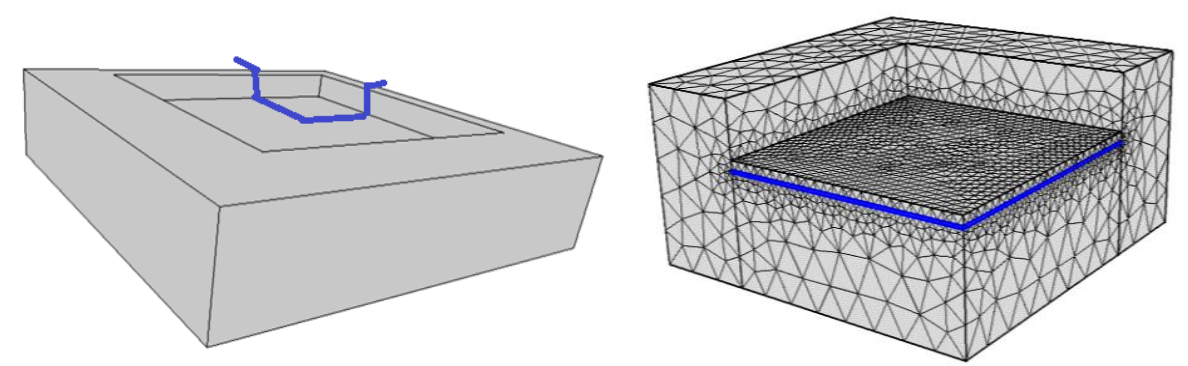

Fig. 2: Simulation model geometry. Left: the symmetric device geometry. Right: only quadrant is modeled

Two sensor structures are selected for simulation, one with the dimension of the square diaphragm is $500 \mu \mathrm{m} \times 500 \mu \mathrm{m}$, the other is $1000 \mu \mathrm{m} \times 1000 \mu \mathrm{m}$. For each structure, the thickness of the diaphragm and the height of isolation gap will be varied. The displacement and capacitance sensitivity will be characterized from the simulation results to find out a set of design parameters giving the best sensor sensitivity.

\subsection{Displacement sensitivity}
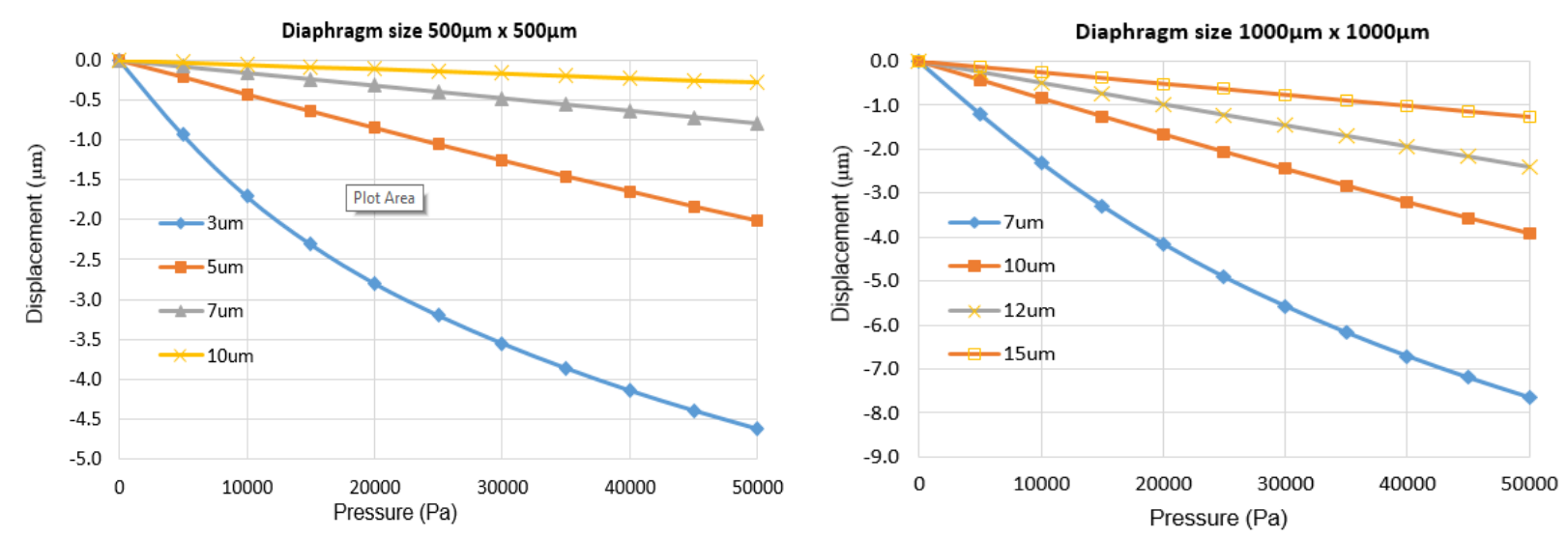

Fig. 3: Centre diaphragm displacement vs applied pressure of two sensor structures on different diaphragm thickness.

The maximum displacement of the sensor diaphragm occurs at the diaphragm centre. At first, the centre diaphragm displacement will be evaluated. The selected diaphragm thickness to be simulated for structure $500 \mu \mathrm{m} \times 500 \mu \mathrm{m}$ includes $3 \mu \mathrm{m}, 5 \mu \mathrm{m}, 7 \mu \mathrm{m}$ and $10 \mu \mathrm{m}$; and for structure $1000 \mu \mathrm{m} \times 1000 \mu \mathrm{m}$ includes $7 \mu \mathrm{m}$, 
$10 \mu \mathrm{m}, 12 \mu \mathrm{m}$ and $15 \mu \mathrm{m}$. Figure 3 illustrates the centre deflection changes vs. applied pressure with the variation of the diaphragm thickness. It can be seen from this figure which diaphragm thickness gives the maximum centre deflection for each sensor structure. For example, with diaphragm size $500 \mu \mathrm{m} \times 500 \mu \mathrm{m}$, the thickness $3 \mu \mathrm{m}$ yields maximum centre deflection, which increases from $1.75 \times 10^{-11} \mathrm{~m}$ at $0 \mathrm{KPa}$ to $4.66 \times 10^{-}$ ${ }^{6} \mathrm{~m}$ at $50 \mathrm{KPa}$. This results in the displacement sensitivity of the sensor (dw/dP) about $93 \mathrm{~nm} / \mathrm{KPa}$. Displacement sensitivities for other set of sensor design parameters are similarly calculated. The displacement of diaphragm has an important effect on the capacitance sensitivity of the sensor.

\subsection{Capacitance sensitivity}

The sensor capacitance output depends on diaphragm dimension and thickness. Generally, diaphragm with larger area and smaller thickness results in larger capacitance output and higher sensitivity. Besides, the gap between plates also plays an important role in calculating the capacitance. However, the diaphragm thickness and the gap should be chosen appropriately to prevent the collapse of diaphragm on bottom electrode. For safe operation, the diaphragm maximum displacement should be smaller than a half of the air gap [12].

Figure 4 presents the change in capacitance output of the sensor versus the applied pressure on different diaphragm thickness for the diaphragm size $500 \mu \mathrm{m}$ x $500 \mu \mathrm{m}$, gap between plates $5 \mu \mathrm{m}$. As illustrated from Figure 4, when pressure applies from 0 to $50 \mathrm{KPa}$, the capacitance of the sensor increases from $110 \mathrm{fF}$ to $235 \mathrm{fF}$ for the diaphragm thickness $3 \mu \mathrm{m}$. So the total variation of the capacitance is $125 \mathrm{fF}$, which yields the capacitance sensitivity $(\mathrm{dC} / \mathrm{dP})$ of $2.48 \mathrm{fF} / \mathrm{KPa}$. In the same way, the capacitance sensitivity, or sensor sensitivity, can be calculated for other diaphragm thickness and for different sensor parameter sets as displayed in Table 1. Table 1 also shows the gaps selected for simulation of each sensor structure.

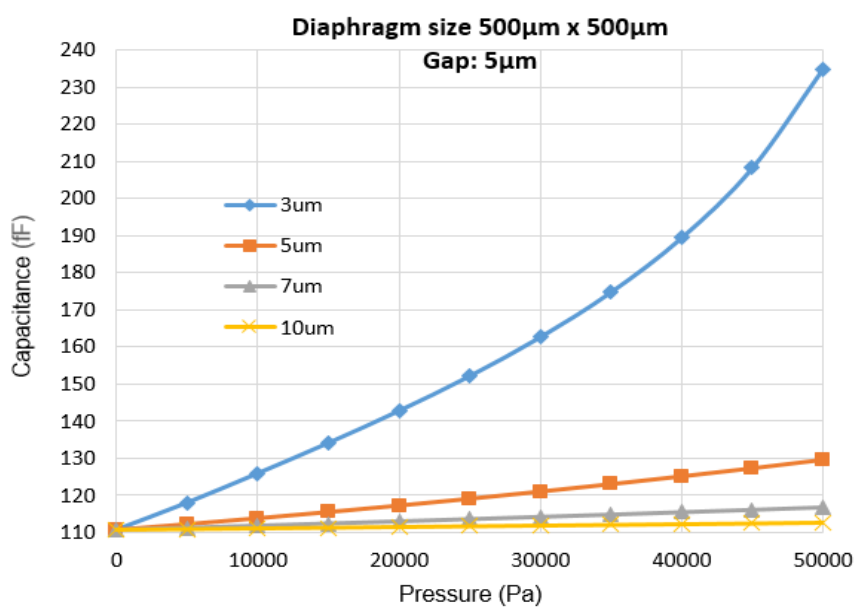

Fig. 4: Capacitance vs. applied pressure on different diaphragm thickness.

Table 1: Capacitance sensitivity for different set of design parameters

\begin{tabular}{|c|c|c|c|c|c|}
\hline \multicolumn{6}{|c|}{ Diaphragm size $500 \mu \mathrm{m} \times 500 \mu \mathrm{m}$} \\
\hline \multirow{2}{*}{\multicolumn{2}{|c|}{$\begin{array}{c}\text { Capacitance } \\
\text { sensitivity } \\
(\mathrm{fF} / \mathrm{KPa})\end{array}$}} & \multicolumn{4}{|c|}{ Diaphragm thickness } \\
\hline & & $3 \mu \mathrm{m}$ & $5 \mu \mathrm{m}$ & $7 \mu \mathrm{m}$ & $10 \mu \mathrm{m}$ \\
\hline \multirow{4}{*}{ Gap } & $3 \mu \mathrm{m}$ & $\mathrm{x}$ & 1.44 & 0.37 & 0.12 \\
\hline & $5 \mu \mathrm{m}$ & 2.48 & 0.38 & 0.12 & 0.04 \\
\hline & $7 \mu \mathrm{m}$ & 0.67 & 0.18 & 0.06 & 0.02 \\
\hline & $10 \mu \mathrm{m}$ & 0.25 & 0.08 & 0.028 & 0.01 \\
\hline
\end{tabular}

\begin{tabular}{|c|c|c|c|c|c|}
\hline \multicolumn{6}{|c|}{ Diaphragm size $1000 \mu \mathrm{m} \times 1000 \mu \mathrm{m}$} \\
\hline \multirow{2}{*}{\multicolumn{2}{|c|}{$\begin{array}{c}\text { Capacitance } \\
\text { sensitivity } \\
(\mathrm{fF} / \mathrm{KPa})\end{array}$}} & \multicolumn{4}{|c|}{ Diaphragm thickness } \\
\hline & & $7 \mu \mathrm{m}$ & $10 \mu \mathrm{m}$ & $12 \mu \mathrm{m}$ & $15 \mu \mathrm{m}$ \\
\hline \multirow{5}{*}{ Gap } & $3 \mu \mathrm{m}$ & $\mathrm{x}$ & $\mathrm{x}$ & 8.52 & 2.66 \\
\hline & $5 \mu \mathrm{m}$ & $\mathrm{x}$ & $\mathrm{x}$ & 1.93 & 0.83 \\
\hline & $8 \mu \mathrm{m}$ & $\mathrm{x}$ & 1.24 & 0.64 & 0.30 \\
\hline & $10 \mu \mathrm{m}$ & $\mathrm{x}$ & 0.72 & 0.38 & 0.19 \\
\hline & $16 \mu \mathrm{m}$ & 0.64 & 0.25 & $1.4 \mathrm{e}-4$ & $7 e-5$ \\
\hline
\end{tabular}

As presented above, the distance between places should be more than twice the maximum diaphragm displacement. With this requirement, capacitance sensitivity values which are written in italic form or by character " $x$ " are not appropriate to use. It can be seen from the table 1 that an increase of the separation 
between the plate's leads to decrease of the capacitance value, as well as the sensitivity. If distance between two plates is low then change in capacitance is high and sensitivity is also high. In general, the high pressure sensitivity of the sensor is achieved by increasing diaphragm size, reducing diaphragm thickness, and decreasing gap between plates. However, a caution is needed when choosing the thickness of diaphragm and the gap for safe sensor operation.

Among the accepted sets of diaphragm thickness and gap, the diaphragm of thickness $5 \mu \mathrm{m}$ and the gap between plates $5 \mu \mathrm{m}$ gives the highest sensor sensitivity which is $0.38 \mathrm{fF} / \mathrm{KPa}$ for diaphragm size $500 \mu \mathrm{m} \mathrm{x}$ $500 \mu \mathrm{m}$. With diaphragm size $1000 \mu \mathrm{m} \times 1000 \mu \mathrm{m}$, values of good capacitance sensitivity can be chosen such as $1.24 \mathrm{fF} / \mathrm{KPa}, 1.93 \mathrm{fF} / \mathrm{KPa}$, but the diaphragm thickness $15 \mu \mathrm{m}$ and the gap $3 \mu \mathrm{m}$ yields the best sensitivity which is $2.66 \mathrm{fF} / \mathrm{KPa}$.

In addition to diaphragm size and thickness, different materials are also selected for diaphragm material, including Silicon, Polysilicon and Silicon carbide. The material properties in table 2 will be used in the analysis and simulation of capacitive pressure sensor model.

Table 2. Physical properties of Si, PolySi and $\mathrm{SiC}$

\begin{tabular}{|c|c|c|c|}
\hline Material Properties & Silicon & Polysilicon & Silicon Carbide \\
\hline Young's Modulus [GPa] & 170 & 160 & 700 \\
\hline Possion's Ratio $(v)$ & 0.28 & 0.22 & 0.18 \\
\hline Density $\left[\mathrm{kg} / \mathrm{m}^{3}\right]$ & 2329 & 2320 & 3200 \\
\hline Relative Permitivity $\left(\varepsilon_{r}\right)$ & 11.7 & 4.5 & 10 \\
\hline
\end{tabular}
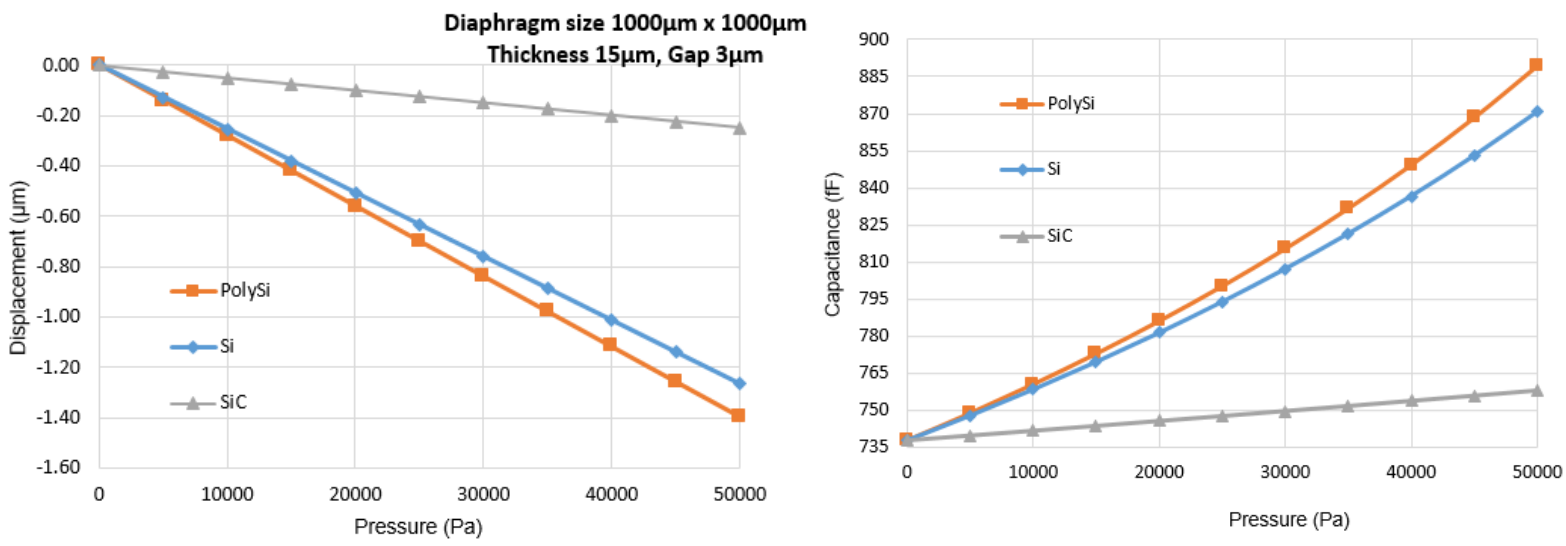

Fig. 5: Centre diaphragm deflection and change in capacitance vs applied pressure for different materials.

The material characterization is performed on sensor diaphragm size $1000 \mu \mathrm{m} \times 1000 \mu \mathrm{m}$, thickness $15 \mu \mathrm{m}$, and gap between plates $3 \mu \mathrm{m}$. From figure 5 with a particular applied pressure, polysilicon and silicon diaphragms provide much higher centre displacement and sensor capacitance compared to $\mathrm{SiC}$ which has higher Young's modulus. This results in higher sensor sensitivity for polysilicon and silicon diaphragm, in which polysilicon diaphragm gives the best sensitivity. Deflection of diaphragm depends on young's modulus, and deflection of diaphragm decreases when young's modulus increases. Based on this, change in capacitance is also low for high Young's modulus materials. It also points out that Polysilicon material is more suitable for low pressure sensing application.

\section{Conclusion}

Some design parameters have been proposed and simulated for the capacitive pressure sensor used in human blood pressure monitor. Capacitive pressure sensor with diaphragm dimension $1000 \mu \mathrm{mx} 1000 \mu \mathrm{m}$, thickness $15 \mu \mathrm{m}$, and the distance between plates $3 \mu \mathrm{m}$ yields the best sensitivity. The polysilicon diaphragm shows high sensitivity on centre deflection and capacitance, on comparing with silicon and silicon carbide. The design also has good linear variation of deflection and capacitance over the working range of pressure.

\section{Acknowledgements}


This research is the output of the project "Investigate, design and simulate a pressure sensor used in blood pressure monitor" under grant number D2014-01 which belongs to University of Information Technology-Vietnam National University HoChiMinh City.

\section{References}

[1] D.S. Eddy, D.R. Sparks. Application of MEMS Technology in Automotive Sensors and Actuators. Proceedings of the IEEE, (1998)

[2] A. Hussian, M.A. Hannan, H. Sanusi, A. Mohamed, B.Y. Majlis. Characterization of MEMS automotive sensor for tire pressure monitoring system. Journal of applied science, pp. 810-815, (2006)

[3] T. Pedersen, G. Fragiacomo, O. Hansen, E.V. Thomsen. Highly sensitive micromachined capacitive pressure sensor with reduced hysteresis and low parasitic capacitance. Sensor and Actuatos A: Physical, Vol 154, No. 1, $35-41,(2009)$

[4] M.X. Zhou, Q.A. Huang, M. Qin, W. Zhou. A novel capacitive pressure sensor based on sandwich structures. Journal of Microelectromechanical Systems, Vol. 14, No. 6, 1272-1282, (2005)

[5] P. Cong, W. H. Ko and D. J. Young. Wireless Batteryless Implantable Blood Pressure Monitoring Microsystem for Small Laboratory Animals. IEEE Sensors Journal, vol. 10, no. 2, pp. 243-254, (2010)

[6] J. A. Potkay. Long term, implantable blood pressure monitoring systems. Biomedical Microdevices 10(3): 379392, (2008)

[7] G. Jiang. Design challenges of implantable pressure monitoring system. Frontiers in neuroscience 4, (2010)

[8] T. Yilmaz, R. Foster, and Y. Hao. Detecting Vital Signs with Wearable Wireless Sensors. Sensors (Basel, Switzerland), 10(12), 10837-10862, (2010)

[9] E. Chung, G. Chen, B. Alexander, M. Cannesson. Non-invasive continuous blood pressure monitoring: a review of current applications." Frontiers of Medicine 7(1): 91-101, (2013)

[10] G.A. Goodall. Design of an implantable micro-scale pressure sensor for managing glaucoma. Michigan State University, Department of Mechanical Engineering, (2002)

[11] S. Timoshenko. S. Woinowsky-Krieger. Theory of Plates and Shells. New York, McGraw-Hill, (1959)

[12] E.V. Thomsen, J. Richter. Piezo Resistive MEMS Devices: Theory and Applications. Department for Micro and Nano Technology, DTU 